\title{
The Two-Point Function and the Effective Magnetic Field in Diluted Ising Models on the Cayley Tree
}

\author{
J. C. A. Barata* \& D. H. U. Marchetti ${ }^{\dagger}$ \\ Instituto de Física \\ Universidade de São Paulo \\ P. O. Box 66318 \\ São Paulo, SP, Brazil
}

\begin{abstract}
Some results on the two-point function and on the analytic structure of the momenta of the effective fugacity at the origin for a class of diluted ferromagnetic Ising models on the Cayley tree are presented.
\end{abstract}

Key words: Lee-Yang singularities; Griffiths' singularities; Two-point functions; Susceptibility.

\section{Contents}

1 Introduction and Previous Results $r$

\begin{tabular}{|lll}
\hline 2 & The Two-Point Correlation Function & 7
\end{tabular}

2.1 The One Point Function . . . . . . . . . . . . . . . . . . . . . . . . . . . . . . . 7

2.2 The Two Point Function $\ldots \ldots \ldots \ldots$

3 The Effective Fugacity and the Analyticity Domain of $\mathcal{M}_{r} \quad 12$

3.1 The Analyticity Domain of $\mathcal{F}_{r} \ldots \ldots \ldots$. . . . . . . . . . . . . . . . . . . . 13

3.2 Estimates on the Location of the Poles of $\tau_{z}^{(n)}(1)$. . . . . . . . . . . . . . . . . . . 17

\begin{tabular}{|lr}
\hline A The Contraction Theorem & 19
\end{tabular}

*e-mail: jbarata@fma.if.usp.br. Partially supported by CNPq.

${ }^{\dagger}$ e-mail: marchett@ime.usp.br. Partially supported by CNPq. 


\section{Introduction and Previous Results}

In a previous work [BM] a detailed analysis of the presence of Griffiths' singularities has been performed in a class of diluted ferromagnetic Ising models on the Cayley tree. This involved the study of the analytic structure of the quenched magnetization at the origin, as a function of the fugacity, as well as the study of its differentiability for real values of the magnetic field. A detailed proof has been presented of the infinite differentiability of the quenched magnetization for real magnetic fields under suitable choices of disorder. The set of Lee-Yang zeros of the deterministic ferromagnetic Ising model on the Cayley tree has been also studied and detailed results have been presented, both in the ferromagnetic and in the paramagnetic phases, showing that this set becomes dense in subsets of the unit circle when the thermodynamic limit is taken. In the ferromagnetic phase, in particular, the set of Lee-Yang zeros becomes dense in the whole unit circle when this limit is performed. These facts are responsible for the emergence of Griffiths' singularities and for the absence of metastable states in the diluted models considered.

In this note we extend our previous results on the diluted models considered. Our main intention here is to present some results on the momenta of the effective fugacity (defined below) produced on the spin at the origin in the random model introduced in [BM], as well as an explicit expression for the two-point functions of that model. Some conclusions on the quenched susceptibility at the origin are drawn.

As in [BM], we consider a random Ising ferromagnet in an homogeneous rooted Cayley tree of order $2, \mathcal{C}_{2}$, described by the Hamiltonian

$$
\mathcal{H}(\sigma ; \xi):=-\sum_{\langle x y\rangle} J_{x y} \sigma_{x} \sigma_{y}-H \sum_{x} \sigma_{x}
$$

where $\sigma: \mathcal{C}_{2} \ni x \longmapsto \sigma_{x} \in\{1,-1\}$ is a configuration of spins. The coupling constants $J_{x y}$ are random variables whose distribution is described as follows. To each generation $M$ of $\mathcal{C}_{2}$ a Bernoulli random variable $\xi_{M}$

$$
\xi_{M}= \begin{cases}1, & \text { with probability } p_{M} \\ 0, & \text { with probability } q_{M}=1-p_{M}\end{cases}
$$

is assigned and we then set $J_{x y}=\xi_{M}$ if $\langle x y\rangle \equiv b$ is a bond at the generation $M$ and 0 otherwise. See Figure 囵.

For any event $A$, which is an element of the $\sigma$-algebra generated by the space of spin configurations, we define the quenched expected value of $A$ as

$$
\bar{A} \equiv \mathbb{E}[A]=\mathbb{E}_{\xi}\langle A\rangle(\xi)
$$

where $\mathbb{E}_{\xi}$ is the expectation with respect to the disorder variables and

$$
\langle A\rangle(\xi)=\frac{1}{Z(\xi)} \sum_{\sigma} A(\sigma) e^{-\beta \mathcal{H}(\sigma ; \xi)}
$$

is the thermal average with

$$
Z(\xi)=\sum_{\sigma} e^{-\beta \mathcal{H}(\sigma ; \xi)}
$$

being the partition function. 


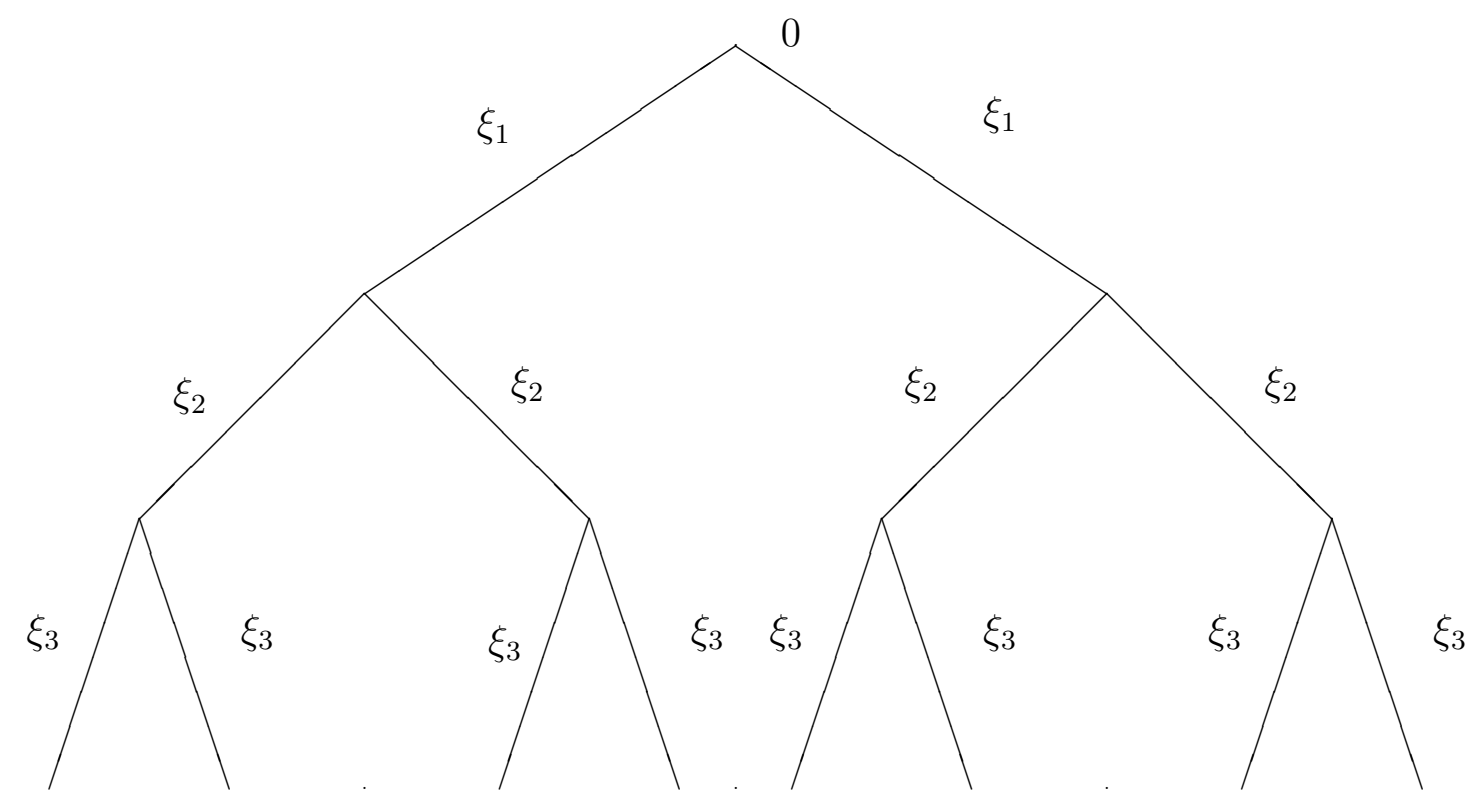

Figure 1: The homogeneous rooted Cayley tree of order 2 and the couplings $\xi_{M}$.

The expectation (1.3) is a function of the inverse temperature $\beta$, the external magnetic field $H$ and the sequence of Bernoulli parameters $\mathbf{p}=\left\{p_{j}\right\}_{j=1}^{\infty}$. We let $\pi=\left\{\mathbf{p}: 0<p_{j} \leq 1, j=1,2, \ldots\right\}$ and for $0 \leq a \leq 1$, let $\pi_{a}=\left\{\mathbf{p} \in \pi: \lim _{n \rightarrow \infty} p_{1} \ldots p_{n}=a\right\}$. Also, for convenience, we write $\zeta=e^{-2 \beta}$ and $z=e^{-2 \beta H}$, the so-called fugacity.

We first take the expectation in a finite tree $\mathcal{C}_{2, M}, M=1,2, \ldots$, with free boundary conditions and study the function $\bar{A}:[0,1] \times \mathbb{C} \times \pi \longmapsto \bar{A}(\zeta, z, \mathbf{p}) \in \mathbb{C}$ in the limit as $M$ goes to infinite.

In $[\mathbb{B M}]$ we were concerned, following $[\mathbb{G}]$, with the magnetization at the origin $m:=\mathbb{E}\left[\sigma_{0}\right]=$ $\mathbb{E}_{\xi}\left\langle\sigma_{0}\right\rangle(\xi)$ which can be written as

$$
m=F+I
$$

where

$$
F:=\sum_{M=0}^{\infty} a_{M}\left\langle\sigma_{0}\right\rangle_{M}(1)
$$

and

$$
I:=\lim _{N \rightarrow \infty} p_{1} \ldots p_{N}\left\langle\sigma_{0}\right\rangle_{N}(1)
$$

give the contribution to $m$ due to finite clusters and the (unique) infinite cluster, respectively. Here, for any observable $A,\langle A\rangle_{M}$ is the thermal average (1.4) with $\mathcal{C}_{2}$ replaced by the finite tree $\mathcal{C}_{2, M}$ and $a_{M}, M \in \mathbb{N}$, is given by

$$
a_{N}:=p_{1} \ldots p_{N}\left(1-p_{N+1}\right), \quad N \geq 1
$$

with $a_{0}:=1-p_{1}$. Clearly, $\left\{a_{n}\right\}_{n=0}^{\infty}$ is a summable sequence with $\sum_{n=0}^{\infty} a_{n}=1-a$ for $p \in \pi_{a}$. 
Equation (1.5) is a consequence of the fact that $\left\langle\sigma_{0}\right\rangle(\xi)=\left\langle\sigma_{0}\right\rangle_{M}(1)$ for all $\xi$ such that $\xi_{1}=$ $\cdots=\xi_{M}=1$ and $\xi_{M+1}=0$ with the finite volume one-point function given as follows (see Section 2 for details):

$$
\left\langle\sigma_{0}\right\rangle_{M}(1)=\frac{1-z \tau_{z}^{(M)}(1)}{1+z \tau_{z}^{(M)}(1)}
$$

where $\tau_{z}^{(n)}$ denotes the $n$-th composition of $\tau_{z}$ with itself: $\tau_{z}^{(n)}:=\underbrace{\tau_{z} \circ \cdots \circ \tau_{z}}_{n \text {-times }}$ and, for $z \in \mathbb{C}$, $\tau_{z}: \mathbb{C} \mapsto \mathbb{C}$ is a rational map given by $\tau_{z}(u):=h(z u)$, with

$$
h(w):=\left(\frac{\zeta+w}{1+\zeta w}\right)^{2} .
$$

The analytic properties of $F$ and $I$ have been studied in [BM] based on the following facts.

Let us denote by $D_{<1}$ the open unit disk in the Riemann sphere $S^{2}$ and by $D_{>1}$ the complement of its closure. In $\mathbb{B M}]$ it was shown that, for the whole interval $0 \leq \zeta<1$, the function $h$ maps $D_{<1}, D_{>1}$ and $S^{1}$ into itself. This implies that, as function of $z, \tau_{z}^{(n)}(1)$ maps each of the sets $D_{<1}, S^{1}$ and $D_{>1}$ into itself for all $n \in \mathbb{N}$ and all $\zeta \in[0,1)$. In addition, each of the sets $[0,1)$ and $(1, \infty) \subset \mathbb{R}_{+}$is also mapped into itself and, for $z=1$ one has $\tau_{1}^{(n)}(1)=1$, for all $n \in \mathbb{N}$. The description of the Lee-Yang singularities and the smoothness of the quenched magnetization are also obtained from these and further properties of the orbit $\tau_{z}^{(n)}(1), n \in \mathbb{N}$, of the dynamical system induced by $\tau_{z}$. For the present analysis these properties will play an important role as well.

Let $H_{j}$ be the effective magnetic field acting on the spins at generation $j$ and let $\Delta_{j}=e^{-2 \beta H_{j}}$ be the associated effective fugacity. We have the following recurrence relation

$$
\Delta_{j-1}=\tau_{j, z}\left(\Delta_{j}\right), \quad j=1, \ldots, M,
$$

with $\Delta_{M}=1$ and $z \in \mathbb{C}$, where

$$
\tau_{j, z}(u)=h_{j}(z u)
$$

and $h_{j}$ is given by (1.7) with $\zeta$ replaced by $\zeta_{j}=e^{-2 \beta \xi_{j}}$. In this work we will be concerned with the effective fugacity at the origin $\Delta_{0}=\tau_{j, z}^{(M)}(1)$. The reason for this nomenclature comes from the analogy between (1.6) and the expression for the magnetization of a lattice containing a single spin: $m=\frac{1-z}{1+z}$.

Since the couplings are random, so are the quantities $\Delta_{j}$ 's. Note that $\Delta_{j}$ depends on the generation level $M$ of the Cayley tree through its initial condition. The distribution of $\Delta_{0}$, when $M \rightarrow \infty$, is worth studying by its own right. In the next theorem the analytic properties of the moments $\mathcal{M}_{s}=\mathbb{E}_{\xi}\left[\Delta_{0}(\xi)^{s}\right], s \in \mathbb{N}$, of $\Delta_{0}$ will be described. We begin by noting that they can be written as

$$
\mathcal{M}_{s}=\mathcal{F}_{s}+\mathcal{I}_{s},
$$

where $\mathcal{F}_{s}:=\lim _{M \rightarrow \infty} \mathcal{F}_{s, M}$ with

$$
\mathcal{F}_{s, M}:=\sum_{n=0}^{M-1} a_{n}\left(\tau_{z}^{(n)}(1)\right)^{s}
$$


and $\mathcal{I}_{s}:=\lim _{M \rightarrow \infty} \mathcal{I}_{s, M}$ with

$$
\mathcal{I}_{s, M}:=p_{1} \ldots p_{M}\left(\tau_{z}^{(M)}(1)\right)^{s}
$$

Our first result is in the following theorem, which will be proven in Section 3. The proof involves a careful analysis of the dynamical system induced by $\tau_{z}$ on the complex plane.

Theorem 1.1 (Moments) For fixed $s \in \mathbb{N}$, let $\mathcal{M}_{s}: \mathbb{C} \times[0,1] \times \pi \ni(z, \zeta, \mathbf{p}) \longmapsto \mathcal{M}_{s}(z, \zeta, \mathbf{p}) \in$ $\mathbb{C}$ be given by (1.10). Then $\mathcal{M}_{s}$ is an analytic function in $z$ for $|z|<1$. In addition, for $\zeta \in[0,1]$, $\mathcal{M}_{s}$ is a holomorphic function of $z$ in the open set $\{z \in \mathbb{C} ;|z|>1\} \backslash \overline{\mathcal{Z}}$, where $\overline{\mathcal{Z}}$ is the closure of

$$
\mathcal{Z}:=\bigcup_{a \in \mathbb{N}} \mathcal{Z}_{a}
$$

with $\mathcal{Z}_{a}$ being the set of the $2^{a+1}-1$ solutions of the equation

$$
\underbrace{\tau_{z} \circ \cdots \circ \tau_{z}}_{a \text {-times }}(1)+\frac{1}{\zeta z}=0 .
$$

Furthermore, $\mathcal{Z}_{a}$ are self-conjugated sets (i.e., $\bar{z} \in \mathcal{Z}_{a}$ if $z \in \mathcal{Z}_{a}$ ) satisfying $\mathcal{Z}_{a} \cap[0, \infty)=\emptyset$ and $\mathcal{Z}^{0} \equiv \overline{\mathcal{Z}} \backslash \mathcal{Z} \subset S^{1}$. There exists $a \zeta_{0} \in[0,1 / 3]$ with $\zeta_{0} \simeq 0.29559$ 円 such that, for $\zeta \in\left[0, \zeta_{0}\right)$, we have the inclusion

$$
\mathcal{Z}_{a} \subset\left\{z \in \mathbb{C}: 1<|z|<(1 / \zeta)^{1 /(a+1)}\right\}
$$

We conjecture that $\mathcal{Z}^{0}=S^{1}$ for $\zeta \in[0,1 / 3]$.

The elements of the set $\mathcal{Z}_{a}$ are poles of $\tau_{z}^{(a+1)}(1)$. These singularities are removable in the magnetization (1.6). Theorem 1.1 states that the set of singular points of $\mathcal{M}_{s}$ is located in $D_{>1}$ and accumulate on a subset $\mathcal{Z}^{0}$ of $S^{1}$. Figure 2 describes the singular set $\mathcal{Z}$. We conjecture that the accumulation set $\mathcal{Z}^{0}$ coincides with the accumulation set of the Lee-Yang singularities of the magnetization at the origin, studied in [BM]. Numerical computations seem to confirm this idea. The inclusion (1.13) indicates how fast the sets $\mathcal{Z}_{a}$ tend to accumulate on $S^{1}$ for $\zeta \in\left[0, \zeta_{0}\right)$.

As a consequence of the recurrence relation (1.8), we are also able to compute the two-point function explicitly. The next result shows that the fluctuation on the Cayley tree is bounded by that of the one-dimensional lattice model.

Theorem 1.2 (Two-Point Function) The truncated two-point function

$$
\left\langle\sigma_{0} ; \sigma_{x}\right\rangle_{N}(\xi):=\left\langle\sigma_{0} \sigma_{x}\right\rangle_{N}(\xi)-\left\langle\sigma_{0}\right\rangle_{N}(\xi)\left\langle\sigma_{x}\right\rangle_{N}(\xi)
$$

can be written as

$$
\left\langle\sigma_{0} ; \sigma_{x}\right\rangle_{N}(\xi)=s\left(z \Delta_{0}\right) \prod_{j=1}^{n} t\left(\zeta_{j}, z \Delta_{j}\right)
$$

where $n=n(x)=\operatorname{dist}(0, x)$ is the generation of the site $x$,

$$
s(x):=1-\left(\frac{1-x}{1+x}\right)^{2}
$$

${ }^{1}$ See also Remark 3.13. 


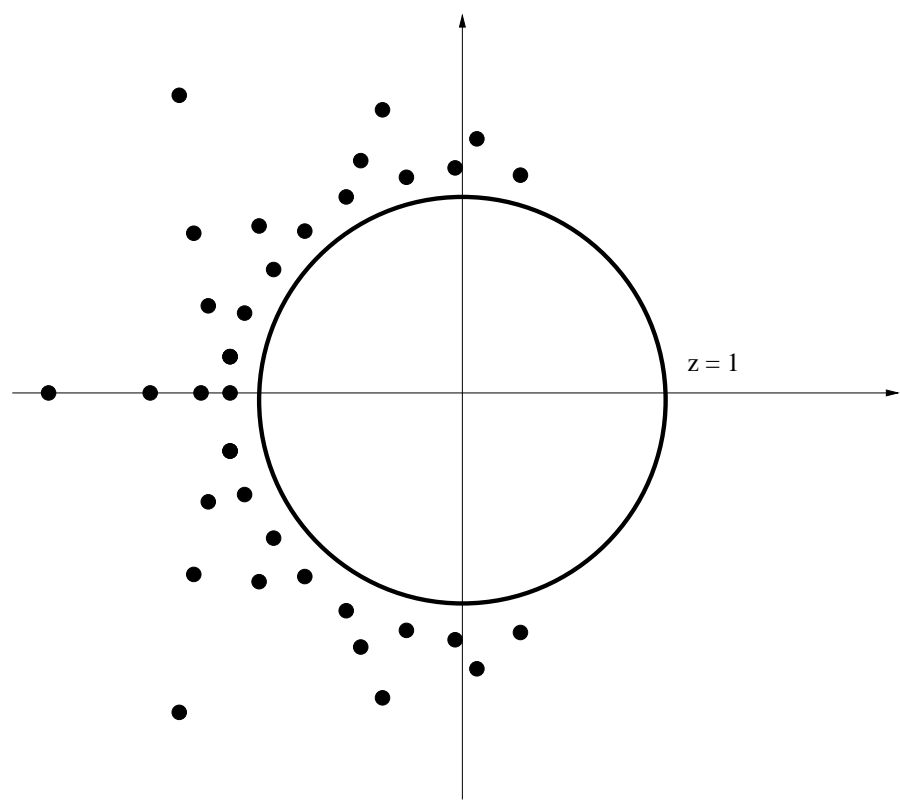

Figure 2: The location of the set of poles $\mathcal{Z}$ and the unit circle $S^{1}$. The points of $\mathcal{Z}$ accumulate on subsets $\mathcal{Z}^{0}$ of the unit circle. Numerical computations indicate that $\mathcal{Z}^{0}=S^{1}$ in the ferromagnetic phase.

and

$$
t(x)=t(\zeta, x):=\frac{\zeta^{-1}-\zeta}{x^{-1}+x+\zeta^{-1}+\zeta} .
$$

For all $\zeta \in[0,1], z \in \mathbb{R}_{+}$and $\mathbf{p} \in \pi_{a}, 0 \leq a \leq 1$, the following identity holds for the expected value $\mathbb{E}_{\xi}\left\langle\sigma_{0} ; \sigma_{x}\right\rangle(\xi)=\lim _{N \rightarrow \infty} \mathbb{E}_{\xi}\left\langle\sigma_{0} ; \sigma_{x}\right\rangle_{N}(\xi)$ :

$$
\mathbb{E}_{\xi}\left\langle\sigma_{0} ; \sigma_{x}\right\rangle(\xi)=\sum_{k=n}^{\infty} a_{k} s\left(w_{k}\right)\left[\prod_{j=1}^{n} t\left(w_{k-j}\right)\right]+a s(w)[t(w)]^{n}
$$

where $w=w(\zeta, z)$ is the limit point of the sequence $w_{n}=z \tau_{z}^{(n)}(1), n \in \mathbb{N}$.

Remark 1.3 A simple upper bound on (1.15) for the paramagnetic phase can be obtained by using Perez's self-avoiding random walk estimate [P]. Only one term, corresponding to the single selfavoiding path connecting 0 to $x$, contributes to the correlation functions in $\mathcal{C}_{k}$. This fact shows that the Ising model on the Cayley tree behaves, on what concerns the asymptotic behavior of the correlations, as a one dimensional system. As a consequence, the correlation length is always finite, even at the transition point. (Note that $|t(\zeta, x)| \leq 1 / 2$ if $\zeta \in[1 / 3,1]$ and $x \in \mathbb{R}_{+}$).

Our last results shows that the quenched susceptibility at the origin $\chi:=\lim _{N \rightarrow \infty} \chi_{N}$ given by

$$
\chi_{N}:=\sum_{x \in \mathcal{C}_{2, N}} \mathbb{E}_{\xi}\left\langle\sigma_{0} ; \sigma_{x}\right\rangle(\xi)
$$


diverges as $\left|\zeta-\zeta_{c}\right|^{\gamma}$ at the critical point $\zeta_{c}=1 / 3$ with an exponent given by the classical theory $\gamma=1$. More precisely

Theorem 1.4 (Susceptibility) The quenched susceptibility at origin $\chi=\chi(\zeta, z)$ is finite for all $\zeta \in[0,1], z \in \mathbb{R}_{+} \backslash\{1\}$ and $\mathbf{p} \in \pi$. Moreover, $\lim _{z \rightarrow 1} \chi$ is also finite provided $\zeta \neq \zeta_{c}$.

In addition, for $\mathbf{p} \in \pi_{a}, 0<a \leq 1$, the following asymptotic behavior

$$
\chi(\zeta, 1) \sim C_{\eta}|\eta|^{\gamma} \quad \text { as } \quad \eta:=\zeta-\zeta_{\mathrm{c}} \rightarrow 0
$$

holds with $\gamma=1, \lim _{\eta \backslash 0} C_{\eta}=4 a / 3$ and $\lim _{\eta{ }_{0}} C_{\eta}=2 a / 3$ provided the condition

$$
\sum_{n=0}^{\infty} n a_{n}<\infty
$$

is satisfied.

The proofs of Theorems 1.2 and 1.4 will be given in Section 2 .

\section{The Two-Point Correlation Function}

This section is dedicated to the proof of Theorems 1.2 and 1.4. We shall consider the truncated two-point function

$$
\left\langle\sigma_{0} ; \sigma_{x}\right\rangle_{M}(\xi)=\left\langle\sigma_{0} \sigma_{x}\right\rangle_{M}(\xi)-\left\langle\sigma_{0}\right\rangle_{M}(\xi)\left\langle\sigma_{x}\right\rangle_{M}(\xi)
$$

where

$$
\left\langle\sigma_{0} \sigma_{x}\right\rangle_{M}(\xi)=\frac{1}{Z_{M}(\xi)} \sum_{\sigma} \sigma_{0} \sigma_{x} e^{-\beta H(\sigma ; \xi)}
$$

for all $x \in \mathcal{C}_{2, M}$ with $M$ large enough.

\subsection{The One Point Function}

Let us first recall the iteration procedure leading to expression (1.6) for the magnetization at the origin. We start by computing the partition function $Z_{M}(\xi)$ in a finite tree with $M$ generations.

Let $Z_{j}=\left(Z_{j}^{+}, Z_{j}^{-}\right), j=0,1, \ldots, M$, be a sequence of two-component vectors defined recursively by

$$
\begin{aligned}
Z_{j-1}^{\sigma} & :=\left(\sum_{\sigma^{\prime}= \pm 1} e^{\beta \xi_{j} \sigma \sigma^{\prime}} e^{\beta h \sigma^{\prime}} Z_{j}^{\sigma^{\prime}}\right)^{2} \\
& =\left(\zeta_{j} z\right)^{-1}\left(\zeta_{j}^{(1-\sigma) / 2} Z_{j}^{+}+\zeta_{j}^{(1+\sigma) / 2} z Z_{j}^{-}\right)^{2}
\end{aligned}
$$

with $Z_{M}^{+}=Z_{M}^{-}=1$. 
If the spin configurations are summed starting from the branches towards the root, the partition function $Z_{M}(\xi)$ can be written as

$$
Z_{M}(\xi)=z^{-1 / 2} Z_{0}^{+}+z^{1 / 2} Z_{0}^{-}
$$

To compute the one point-function

$$
\left\langle\sigma_{0}\right\rangle_{M}(\xi)=\frac{1}{Z_{M}(\xi)} \sum_{\sigma} \sigma_{0} e^{-\beta H(\sigma ; \xi)},
$$

we repeat the procedure leading to (2.4) for the numerator in (2.5). Except by the last summation on the spin at the root, all remaining ones give exactly the previous expressions. We thus have

$$
\left\langle\sigma_{0}\right\rangle_{M}(\xi)=\frac{z^{-1 / 2} Z_{0}^{+}-z^{1 / 2} Z_{0}^{-}}{z^{-1 / 2} Z_{0}^{+}+z^{1 / 2} Z_{0}^{-}}=\frac{1-z \Delta_{0}}{1+z \Delta_{0}}
$$

where $\Delta_{j}:=Z_{j}^{-} / Z_{j}^{+}$.

From (2.3), we have

$$
\Delta_{j-1}=\left(\frac{\zeta_{j}+z \Delta_{j}}{1+\zeta_{j} z \Delta_{j}}\right)^{2}=\tau_{j, z}\left(\Delta_{j}\right)
$$

and the sequence $\left\{\Delta_{j}\right\}_{j=0}^{M}$ satisfies the recurrence relation (1.8) with $\Delta_{M}=1$. Recall that $\Delta_{j}$ is a random variable since $\zeta_{j}=e^{-2 \beta \xi_{j}}$ with $\xi_{j}$ as given by (1.2).

\subsection{The Two Point Function}

To compute the numerator of (2.2), we repeat the steps in the calculation of the partition function $Z_{M}(\xi)$. Our aim is to derive an expression analogous to (2.4).

Let $\widetilde{Z}_{j}=\left(\widetilde{Z}_{j}^{+}, \widetilde{Z}_{j}^{-}\right), j=0, \ldots, M$, be a sequence of two-component vectors defined recursively as in the following.

For $j=n_{0}+1, \ldots, M$, with $n_{0}=\operatorname{dist}(0, x)$, we have $\widetilde{Z}_{j}^{\sigma}=Z_{j}^{\sigma}$, i.e., $\widetilde{Z}_{j}^{\sigma}$ satisfy the equation (2.3) with initial conditions $\widetilde{Z}_{M}^{+}=\widetilde{Z}_{M}^{-}=1$.

For $j \leq n_{0}$, we consider a linear transformation of the form

$$
\widetilde{Z}_{j-1}^{\sigma}=Z_{j-1}^{\sigma} \frac{\zeta^{(1-\sigma) / 2} \widetilde{Z}_{j}^{+}+\zeta^{(1+\sigma) / 2} z \widetilde{Z}_{j}^{-}}{\zeta^{(1-\sigma) / 2} Z_{j}^{+}+\zeta^{(1+\sigma) / 2} z Z_{j}^{-}}
$$

with $\widetilde{Z}_{n_{0}}^{\sigma}=\sigma Z_{n_{0}}^{\sigma}$.

We now observe that the sum over all spin configurations in the numerator of (2.2), except by spin at the origin, is determined by (2.7) and the sum over all spin configurations in the denominator is determined by (2.3). The two point function (2.2) can thus be written in the following form

$$
\left\langle\sigma_{0} \sigma_{x}\right\rangle_{M}(\xi)=\frac{\widetilde{Z}_{0}^{+}-z \widetilde{Z}_{0}^{-}}{Z_{0}^{+}+z Z_{0}^{-}} .
$$

In the following lemma the equation (2.8) will be reorganized and reexpressed in terms of the quantities $Z_{j}^{\sigma}, j=0,1, \ldots, M$ and $\sigma=+,-$. 
Lemma 2.1 The sequence of vectors $\widetilde{Z}_{n}, n=1, \ldots, n_{0}$, defined by (2.7), can be written as

$$
\widetilde{Z}_{n-1}^{\sigma}=\left(\mathcal{A}_{n}+\sigma \mathcal{B}_{n}\right) Z_{n-1}^{\sigma}
$$

where

$$
\mathcal{A}_{n}=A_{n} \mathcal{B}_{n+1}+\ldots+A_{n_{0}-1} \mathcal{B}_{n_{0}}+A_{n_{0}}
$$

and

$$
\mathcal{B}_{j}=B_{j} B_{j+1} \ldots B_{n_{0}}
$$

with $j=n, \ldots, n_{0}$. Here $A_{j}=A_{j}\left(\zeta_{j}, z\right)$ and $B_{j}=B_{j}\left(\zeta_{j}, z\right), j=1, \ldots, n_{0}$, are random variables given by

$$
A_{j}=\frac{\left(z \Delta_{j}\right)^{-1}-z \Delta_{j}}{\left(z \Delta_{j}\right)^{-1}+z \Delta_{j}+\zeta_{j}^{-1}+\zeta_{j}}
$$

and

$$
B_{j}=\frac{\zeta^{-1}-\zeta}{\left(z \Delta_{j}\right)^{-1}+z \Delta_{j}+\zeta_{j}^{-1}+\zeta_{j}} .
$$

Proof. We shall prove Lemma 2.1 by induction. We let $j=n_{0}$ and observe that, by multiplying the numerator and the denominator of (2.7) by $\left(\zeta_{j}^{-(1-\sigma) / 2}\left(z \Delta_{n_{0}}\right)^{-1}+\zeta_{j}^{-(1+\sigma) / 2}\right) / Z_{n_{0}}^{+}$, it can be written as

$$
\begin{aligned}
\frac{\tilde{Z}_{n_{0}-1}^{\sigma}}{Z_{n_{0}-1}^{\sigma}} & =\frac{\left(z \Delta_{n_{0}}\right)^{-1}-z \Delta_{n_{0}}+\zeta_{j}^{-\sigma}-\zeta_{j}^{\sigma}}{\left(z \Delta_{n_{0}}\right)^{-1}+z \Delta_{n_{0}}+\zeta_{j}^{-\sigma}+\zeta_{j}^{\sigma}} \\
& =\frac{\left(z \Delta_{n_{0}}\right)^{-1}-z \Delta_{n_{0}}+\sigma\left(\zeta_{j}^{-1}-\zeta_{j}\right)}{\left(z \Delta_{n_{0}}\right)^{-1}+z \Delta_{n_{0}}+\zeta_{j}^{-1}+\zeta_{j}} \\
& =A_{n_{0}}+\sigma B_{n_{0}} .
\end{aligned}
$$

Now, let $j=n+1$. Assuming (2.9) valid, (2.7) can be written as

$$
\frac{\tilde{Z}_{n}^{\sigma}}{Z_{n}^{\sigma}}=\mathcal{A}_{n+1}+\frac{\zeta_{j}^{(1-\sigma) / 2}-\zeta_{j}^{(1+\sigma) / 2} z \Delta_{n+1}}{\zeta_{j}^{(1-\sigma) / 2}+\zeta_{j}^{(1+\sigma) / 2} z \Delta_{n+1}} \mathcal{B}_{n+1}
$$

Multiplying the numerator and the denominator of the second term on the right hand side of this equation by $\zeta_{j}^{-(1-\sigma) / 2}\left(z \Delta_{n+1}\right)^{-1}+\zeta_{j}^{-(1+\sigma) / 2}$, gives

$$
\begin{aligned}
\frac{\widetilde{Z}_{n}^{\sigma}}{\widehat{Z}_{n}^{\sigma}} & =\mathcal{A}_{n+1}+\frac{\left(z \Delta_{n+1}\right)^{-1}-z \Delta_{n+1}+\sigma\left(\zeta_{j}^{-1}-\zeta_{j}\right)}{\left(z \Delta_{n+1}\right)^{-1}+z \Delta_{n+1}+\zeta_{j}^{-1}+\zeta_{j}} \mathcal{B}_{n+1} \\
& =\mathcal{A}_{n+1}+\left(A_{n}+\sigma B_{n}\right) \mathcal{B}_{n+1}
\end{aligned}
$$

which, in view of (2.10) and (2.11), concludes the proof of Lemma 2.1. 
Now we proceed with the proof of Theorem 1.2.

Proof of Theorem 1.2. Using (2.9) to compute (2.8), gives

$$
\begin{aligned}
\left\langle\sigma_{0} \sigma_{x}\right\rangle_{M}(\xi) & =\frac{\left(\mathcal{A}_{1}+\mathcal{B}_{1}\right) Z_{0}^{+}-z\left(\mathcal{A}_{1}-\mathcal{B}_{1}\right) Z_{0}^{-}}{Z_{0}^{+}+z Z_{0}^{-}} \\
& =\mathcal{A}_{1} \frac{1-z \Delta_{0}}{1+z \Delta_{0}}+\mathcal{B}_{1}
\end{aligned}
$$

The one-point function at $x$ can be computed by a procedure analogous to one described at the beginning of this subsection. The difference between this one-point function and the two-point function is the spin at origin. As before, (2.7) with $j=0, \ldots, n_{0}$ is of relevance for the description of the numerator of $\left\langle\sigma_{x}\right\rangle$. The iteration gives an expression of the form (2.8) with the minus sign replaced by plus. The one-point function can thus be written as

$$
\left\langle\sigma_{x}\right\rangle_{M}(\xi)=\frac{\widetilde{Z}_{0}^{+}+z \widetilde{Z}_{0}^{-}}{Z_{0}^{+}+z Z_{0}^{-}}=\mathcal{A}_{1}+\mathcal{B}_{1} \frac{1-z \Delta_{0}}{1+z \Delta_{0}} .
$$

Inserting (2.16) and (2.17) into (2.1) and taking into account (2.6), yields

$$
\left\langle\sigma_{0} ; \sigma_{x}\right\rangle_{M}(\xi)=\left[1-\left(\frac{1-z \Delta_{0}}{1+z \Delta_{0}}\right)^{2}\right] \mathcal{B}_{1} .
$$

When the thermodynamic limit, $M \rightarrow \infty$ has been taken, the random variables $\Delta_{j}$ 's in (2.18) can be replaced by their limits $\lim _{M \rightarrow \infty} \Delta_{j}$ (recall that $\mathcal{B}_{1}$ depends on $\left\{\Delta_{j}\right\}_{j=0}^{n_{0}}$, each of which defined by a recursion relation with initial condition $\Delta_{M}=1$ dependent on the generation $\left.M\right)$. Since $\Delta_{j}$ is bounded from above and below, the convergence in distribution is guaranteed by the convergence of their moments $\left[\mathrm{Fe}\right.$. It follows from Theorem 1.1 that $\lim _{M \rightarrow \infty} \mathbb{E}_{\xi} \Delta_{j}$ exists and is a real analytic function of $z$ in $z \in \mathbb{R}_{+} \backslash\{1\}$.

In order to take the expectation value of (2.18) we shall use the following property: for any bounded function $f(\xi)=f\left(\xi_{j}, \xi^{\prime}\right)$ of the random variables $\xi=\left(\xi_{j}, \xi^{\prime}\right)$, we have

$$
\mathbb{E}_{\xi} f(\xi) B_{j}(\xi)=p_{j} \mathbb{E}_{\xi^{\prime}} f\left(1, \xi^{\prime}\right) \frac{\zeta^{-1}-\zeta}{\left(z \Delta_{j}\right)^{-1}+z \Delta_{j}+\zeta^{-1}+\zeta}
$$

since $\zeta_{j}^{-1}-\zeta_{j}=e^{2 \beta \xi_{j}}-e^{-2 \beta \xi_{j}}=0$ if $\xi_{j}=0$.

Define

$$
s(x):=1-\left(\frac{1-x}{1+x}\right)^{2}
$$

and

$$
t(x)=t(\zeta, x):=\frac{\zeta^{-1}-\zeta}{x^{-1}+x+\zeta^{-1}+\zeta} .
$$


Using (2.19) in the expected value of (2.18) gives

$$
\begin{aligned}
\mathbb{E}_{\xi}\left\langle\sigma_{0} ; \sigma_{x}\right\rangle_{M}(\xi) & =p_{1} \ldots p_{n_{0}} \mathbb{E}_{\xi^{\prime}} s\left(z \Delta_{0}\right)\left[\prod_{j=1}^{n_{0}} t\left(z \Delta_{j}\right)\right] \\
& =\sum_{k=n_{0}}^{M-1} a_{k} s\left(w_{k}\right)\left[\prod_{j=1}^{n_{0}} t\left(w_{k-j}\right)\right]+p_{1} \ldots p_{M} s\left(w_{M}\right)\left[\prod_{j=1}^{n_{0}} t\left(w_{M-j}\right)\right]
\end{aligned}
$$

where $w_{n}=z \tau_{z}^{(n)}(1), n \in \mathbb{N}$. Here, we have first taken partial expectations with respect to the variables $\xi_{1}, \ldots, \xi_{n_{0}}$ and have used in the sequel for all remaining expectations that the sequence $\Delta_{j}, j \in \mathbb{N}$, satisfies the recurrence relation $z \Delta_{j-1}=z h_{j}\left(z \Delta_{j}\right)$ with $h_{j}(1)=1$. Equation (1.15) then follows since $s(x)$ and $t(x)$ are continuous in $\mathbb{R}_{+}$and $w_{n}$ converges to the solution $w=w(\zeta, z)$ of the fixed point equation $w=z h(w)$ in this domain provided $z \in \mathbb{R}_{+}$. This concludes the proof of Theorem 1.2.

We now turn to the proof of Theorem 1.4 on the quenched susceptibility at origin $\chi$. We note the following facts on the function $s$ and $t$ (the proof will be omitted):

Proposition 2.2 The function $s: w \in \mathbb{R}_{+} \longmapsto s(w) \in \mathbb{R}_{+}$and $t:(\zeta, w) \in[0,1] \times \mathbb{R}_{+} \longmapsto$ $t(\zeta, w) \in \mathbb{R}_{+}$given by (2.20) and (2.21), respectively, have a maximum value at $w=1$ with $s(1)=1$ and $t(\zeta, 1)=(1-\zeta) /(1+\zeta)$, are monotonically increasing function of $w$ in $[0,1]$ and satisfy $s(w)=s\left(w^{-1}\right)$ and $t(\zeta, w)=t\left(\zeta, w^{-1}\right)$.

For $z \in[0,1)$ we recall that $w_{n}, n \in \mathbb{N}$, is a monotonically decreasing sequence with $w_{n}<1$ and for each $n, w_{n}=w_{n}(z)$ is monotonically decreasing function of $z$ in this domain. So, in view of Proposition 2.2,

$$
2 t\left(\zeta, w_{n}\right) \leq 2 \frac{1-\zeta}{1+\zeta}<1
$$

holds for all $\zeta \in(1 / 3,1]$ and $z \in \mathbb{R}_{+} \backslash\{1\}$.

In addition, for $\zeta \in[0,1 / 3)$ (ferromagnetic phase) and $z \in[0,1), w_{n}$ converges to a nontrivial solution $w(\zeta, z)$ of $w=z h(w)$ (see [BM]) with $w \nearrow \underline{w}$ as $z \nearrow 1$ with

$$
\underline{w}=\frac{1-2 \zeta-\zeta^{2}}{2 \zeta^{2}}-\frac{1-\zeta^{2}}{2 \zeta^{2}}\left(\frac{1-3 \zeta}{1+\zeta}\right)^{1 / 2} .
$$

This and Proposition 2.2 implies that there exist finite number $n_{0}=n_{0}(\zeta, z) \in \mathbb{N}$ such that for $n>n_{0}$

$$
2 t\left(\zeta, w_{n}\right) \leq 2 t(\zeta, \underline{w})=\frac{2 \zeta}{1-\zeta}<1
$$

holds for all $\zeta \in[0,1 / 3)$ and $z \in \mathbb{R}_{+} \backslash\{1\}$. 
Substituting equation (1.15) into (1.16) and taking into account the inequalities (2.23) and (2.24), gives

$$
\begin{aligned}
\chi & =\sum_{n=0}^{\infty} 2^{n} \sum_{k=n}^{\infty} a_{k} s\left(w_{k}\right)\left[\prod_{j=1}^{n} t\left(w_{k-j}\right)\right]+a s(w) \sum_{n=0}^{\infty}[2 t(w)]^{n} \\
& =\sum_{k=0}^{\infty} a_{k} s\left(w_{k}\right) \sum_{n=0}^{k} 2^{n}\left[\prod_{j=1}^{n} t\left(w_{k-j}\right)\right]+\frac{a s(w)}{1-2 t(w)} .
\end{aligned}
$$

Note that

$$
\lim _{z \rightarrow 1} \frac{a s(w)}{1-2 t(w)} \sim C_{\eta}|\eta| \quad \text { as } \quad \eta:=\zeta-\zeta_{c} \rightarrow 0
$$

holds with $\lim _{\eta \searrow 0} C_{\eta}=4 a / 3$ and $\lim _{\eta}{ }_{0} C_{\eta}=2 a / 3$. Note also that, for $\mathbf{p} \in \pi_{a}, 0<a \leq 1$, such that $\sum_{n} n a_{n}<\infty$, the first term on the second line of 2.25) does not diverge at $z=1$ for any $\zeta \in[0,1]$ since, by the dominated convergence theorem,

$$
\lim _{x \nearrow 1} \sum_{k=0}^{\infty} \frac{1-x^{k}}{1-x} a_{k}<\infty .
$$

A non classical exponent $\gamma=\gamma(\mathbf{p})$ may appear if (1.18) is violated.

This concludes the proof of Theorem 1.4.

\section{The Effective Fugacity and the Analyticity Domain of $\mathcal{M}_{r}$}

In this section we will prove Theorem 1.1 concerning the analyticity domain of $\mathcal{M}_{r}$. We split the proof in two parts, the first, and simplest, dedicated to the function $\mathcal{I}_{r}$ and the second, and more elaborated, to the function $\mathcal{F}_{r}$. The study of the singularities of $\mathcal{F}_{r}$ involves a careful analysis of the dynamical system induced by $\tau_{z}$ on the complex plane.

Let us first establish the analyticity results on the functions $\mathcal{I}_{r}(z), r \in \mathbb{N}$.

Let us denote by $D_{<1}$ the open unit disk in $S^{2}$ and by $D_{>1}$ the complement of its closure. More generally, for $a>0$ call

$$
\begin{aligned}
& D_{<a}:=\left\{w \in S^{2}:|w|<a\right\}, \\
& D_{>a}:=\left\{w \in S^{2}:|w|>a\right\} .
\end{aligned}
$$

For further purposes define also for $a, b \in \mathbb{R}_{+}, a<b$,

$$
D_{a, b}:=\left\{w \in S^{2}: a<|w|<b\right\} .
$$

The following theorem has been proven in [BM]: 
Theorem 3.1 The sequence $\tau_{z}^{(n)}(1), n \in \mathbb{N}$, of analytic functions on $D_{<1}$ converges uniformly to an analytic function $\tau=\tau(z)$ on the whole set $D_{<1}$. For all $\zeta \in(0,1]$ the function $\tau=\tau(z)$ fulfills the fixed point equation $\tau=h(z \tau)$ on the whole set $D_{<1}$. As a consequence, $\tau(z)$ has no zeros on $D_{<1}$.

Analyticity of $\mathcal{I}_{r}(z)$ on $D_{<1}$ is, actually, the statement of Theorem 3.1. Let us now consider $\mathcal{I}_{r}(z)$ on $D_{>1}$. For $r \in \mathbb{N}, \mathbf{p} \in \pi_{a}$ and $z \in D_{>1}$, we have

$$
\mathcal{I}_{r}(z)=a \lim _{n \rightarrow \infty}\left[\tau_{z}^{(n)}(1)\right]^{r}=a \lim _{n \rightarrow \infty}\left[\tau_{z^{-1}}^{(n)}(1)\right]^{-r}=a\left[\tau\left(z^{-1}\right)\right]^{-r} .
$$

Therefore, in view of Theorem 3.1 which says that $\tau(w)$ has no zeros for $w \in D_{<1}, \mathcal{I}_{r}(z)$ is an analytic function on $D_{>1}$.

\subsection{The Analyticity Domain of $\mathcal{F}_{r}$}

In this subsection we will establish the analyticity results on the functions $\mathcal{F}_{r}(z), r \in \mathbb{N}$. The proof will be given in two theorems, according to whether $z \in D_{<1}$ or $z \in D_{>1}$.

Let us prove our first result on the analyticity of $\mathcal{F}_{r}(z)$.

Theorem 3.2 (The First Analyticity Theorem) The function $\mathcal{F}_{r}(z)$ defined in (1.11) is an analytic function of $z$ in the open set $D_{<1}$ for all $\mathbf{p} \in \pi$ and $\zeta \in[0,1)$.

Proof. The partial sums

$$
\mathcal{F}_{r, N}(z):=\sum_{n=0}^{N} a_{n}\left(\tau_{z}^{(n)}(1)\right)^{r}, \quad r, N \in \mathbb{N},
$$

are analytic in $D_{<1}$ since the pole of $h$ lies in $D_{>1}$. Moreover, one has $\left|\tau_{z}(1)\right|<1$ for $z \in D_{<1}$ and so, for any $\varepsilon>0$ one has

$$
\left|\mathcal{F}_{r, N}(z)-\mathcal{F}_{r, M}(z)\right|<\sum_{n=N+1}^{M} a_{n} \leq \varepsilon,
$$

for all $N$ and $M, N<M$, large enough, since the sequence $\left\{a_{n}, n \in \mathbb{N}\right\}$ is summable. So, the partial sums form a uniform Cauchy sequence of analytic functions whose limit exists and is analytic in $D_{<1}$.

We will now prove the analyticity of the function $\mathcal{F}_{r}(z)$ in $D_{>1} \backslash \mathcal{Z}$. We start by studying the location of the singularities of $\mathcal{F}_{r}(z)$. First we prove the following lemma.

Lemma 3.3 Let $\mathcal{B} \subset \mathbb{C}$ be an open set such that $\tau_{z}^{(n)}(1), n \in \mathbb{N}$, are analytic on $\mathcal{B}$ and

$$
\inf _{z \in \mathcal{B}} \inf _{n \in \mathbb{N}}\left|\tau_{z}^{(n)}(1)-\left(\frac{-1}{z \zeta}\right)\right|>0 .
$$

Then $\mathcal{F}_{r}(z)$ is an analytic function in $\mathcal{B}$. 
Remark 3.4 Note that $h$ given by (1.7) has a unique double pole at $w=-1 / \zeta$. Moreover, $h(w)$ is bounded on $\mathbb{C} \backslash \mathcal{O}$, where $\mathcal{O}$ is any open set containing the pole.

Remark 3.5 Note that $\inf _{n \in \mathbb{N}}\left|\tau_{z}^{(n)}(1)-\left(-z^{-1} / \zeta\right)\right|=0$ is not a necessary condition for $\mathcal{F}_{r}$ to be singular at $z$. For instance, for $z=-1$, one has $\left|\tau_{z}^{(n)}(1)-\left(-z^{-1} / \zeta\right)\right|=1 / \zeta-1$ for all $n \in \mathbb{N}$. However, as we will see below, $\mathcal{F}_{r}(z)$ is not analytic at $z=-1$ because -1 is an accumulation point of poles of $\mathcal{F}_{r}$.

Proof of Lemma 3.3. If condition (3.6) holds for a given $\mathcal{B}$ it implies that there exists an open neighborhood $\mathcal{O}$ of $-1 / \zeta$ such that, for all $z \in \mathcal{B}$ and for all $n \in \mathbb{N}, z \tau_{z}^{(n)}(1) \in \mathbb{C} \backslash \mathcal{O}$. Since $\tau_{z}^{(n+1)}(1)=h\left(z \tau_{z}^{(n)}(1)\right)$, this means that there exists a positive constant $M$, such that for all $z \in \mathcal{B}$ and all $n \in \mathbb{N},\left|\tau_{z}^{(n)}(1)\right| \leq M$. Hence, by the same argument used in the proof of Theorem 3.2 , the partial sums $\mathcal{F}_{r, N}(z)$ form a uniform Cauchy sequence of analytic functions in $\mathcal{B}$ and consequently, their limit exists and is analytic in $\mathcal{B}$.

Our task now is to localize the points $z \in \mathbb{C}$ where the condition

$$
\inf _{n \in \mathbb{N}}\left|\tau_{z}^{(n)}(1)-\left(\frac{-1}{z \zeta}\right)\right|=0
$$

holds. The next proposition shows that, for each $n \in \mathbb{N}$, there exist at least one point $z \in \mathbb{C}$ such that $\left|\tau_{z}^{(n)}(1)-\left(-z^{-1} / \zeta\right)\right|=0$.

Proposition 3.6 For each $n \in \mathbb{N}$ there is at least one and at most $2^{n+1}-1$ distinct solutions in $\mathbb{C}$ of the equation

$$
\tau_{z}^{(n)}(1)-\left(\frac{-1}{z \zeta}\right)=0 .
$$

Proof. We begin by observing that, $\tau_{z}^{(n)}(1)$ is the ratio of two polynomials of degree $2^{n+1}-2$ : $\tau_{z}^{(n)}(1)=P_{n}(z) / Q_{n}(z)$. This is a consequence of the fact that the function $h$ is the ratio of two polynomials in $z$ of degree 2 . To be more explicit, $P_{n}$ and $Q_{n}$ are defined recursively through

$$
\begin{aligned}
P_{n+1}(z):=\left(\zeta Q_{n}(z)+z P_{n}(z)\right)^{2}, & & n \in \mathbb{N}, \\
Q_{n+1}(z):=\left(Q_{n}(z)+\zeta z P_{n}(z)\right)^{2}, & & n \in \mathbb{N}
\end{aligned}
$$

with $P_{0}(z)=Q_{0}(z):=1$.

Therefore, (3.8) means that

$$
z P_{n}(z)+\frac{1}{\zeta} Q_{n}(z)=0
$$

which has at least one and at most $2^{n+1}-1$ possible distinct solutions in $\mathbb{C}$, since the left hand side is a polynomial of degree $2^{n+1}-1$. Clearly, for $n=0$, the unique solution is the pole of $h$, $z=-1 / \zeta$. 
Let us denote by $\mathcal{Z}_{n}$ the finite set of all $z$ 's satisfying (3.8) for $n \in \mathbb{N}$. Define

$$
\mathcal{Z}:=\bigcup_{a \in \mathbb{N}} \mathcal{Z}_{a}
$$

and let $\overline{\mathcal{Z}}$ be the closure of $\mathcal{Z}$. The set difference $\mathcal{Z}^{0}:=\overline{\mathcal{Z}} \backslash \mathcal{Z}$ is the set of accumulation points of $\mathcal{Z}$.

To establish that $\mathcal{F}_{r}$ is holomorphic in $\mathbb{C} \backslash \overline{\mathcal{Z}}$ we need to describe where the elements of $\mathcal{Z}$ and $\mathcal{Z}^{0}$ are located.

Theorem 3.7 For all $\zeta \in(0,1)$ and $k \in \mathbb{N}$ one has $\mathcal{Z}_{k} \subset D_{>1}$ with the accumulation points of $\mathcal{Z}$ lying in the unit circle, i. e., $\mathcal{Z}^{0} \subset S^{1}$.

In addition, $\mathcal{Z}_{k} \cap[1, \infty)=\emptyset$, for all $k \in \mathbb{N}$. The sets $\mathcal{Z}_{k}$ are also self-conjugated in the sense that $\bar{z} \in \mathcal{Z}_{k}$ if $z \in \mathcal{Z}_{k}$ and, for all $k \in \mathbb{N}$, one has $\mathcal{Z}_{k} \cap(-\infty,-1) \neq \emptyset$. This last fact together with $\mathcal{Z}^{0} \subset S^{1}$ implies that $-1 \in \mathcal{Z}^{0}$.

Proof. Firstly, observe that the equation

$$
\tau_{z}^{(k)}(1)=-\frac{1}{z \zeta}
$$

has no solutions for $z \in D_{1}:=D_{<1} \cup S^{1}$, since $\tau_{z}^{(k)}(1)$ maps $D_{1}$ into itself and $\zeta<1$. This equation has no solutions for $|z| \geq 1 / \zeta$ either, since in this region, the left hand side of (3.11) lies in $D_{>1}$, but the right hand side in $D_{1}$. We conclude that $\mathcal{Z} \subset D_{1,1 / \zeta}$. Analogously, (3.11) has no solutions in $(1, \infty)$, since there its left hand side is positive while the right hand side is negative.

To prove that $\mathcal{Z}^{0} \subset S^{1}$ we proceed as follows. The elements of $\mathcal{Z}_{n}$ are the poles of $\tau_{z}^{(n+1)}(1)$ in $D_{>1}$. Therefore, if $w \in \mathcal{Z}_{n}, w^{-1}$ is a zero of $\tau_{z}^{(n+1)}(1)$ in $D_{<1}$. The sequence of functions $\tau_{z}^{(n)}$ converges uniformly in $D_{<1}$ to an analytic function $\tau=\tau(z)$ (Theorem 3.1). According to a theorem by Hurwitz (see, e.g., [T]) the fact that $\tau$ has no zeros on $D_{<1}$ (Theorem 3.1) implies that the set of zeros of the functions $\tau_{z}^{(n)}$ has no limit points on $D_{<1}$ and therefore, if they exist, they must lie on $S^{1}$.

The sets $\mathcal{Z}_{k}$ are all self-conjugated by the definition of $h$, since the left hand side of (3.10) is a polynomial with real coefficients. This polynomial is also of odd degree, namely $2^{k+1}-1$. Both facts together imply that this polynomial has at least one real root. However, according to the previous remarks, the real roots cannot lie in $[-1, \infty)$. So, they must be negative and lower than -1 . From the fact that $\mathcal{Z}^{0} \subset S^{1}$ it follows that these negative roots must converge to -1 when $k \rightarrow \infty$ and so $-1 \in \mathcal{Z}^{0}$. This shows, in particular, that $\mathcal{Z}^{0} \neq \emptyset$.

Remark 3.8 Numerical computations seem to indicate that $\mathcal{Z}^{0}=S^{1}$ for the whole ferromagnetic region $0 \leq \zeta<1 / 3$. Unfortunately we were not able to prove this conjecture.

After these considerations on the location of the singularities of $\tau_{z}^{(n)}$ we turn to the proof of the analyticity statement on $\mathcal{F}_{r}$. 
Lemma 3.9 Let $\mathcal{B}$ be an open set such that $\overline{\mathcal{B}} \subset D_{>1} \backslash \overline{\mathcal{Z}}$, where $\overline{\mathcal{B}}$ is the closure of $\mathcal{B}$. Then the sequence $\tau_{z}^{(n)}(1), n \in \mathbb{N}$, converges to the function $1 / \tau\left(z^{-1}\right)$ uniformly on $\mathcal{B}$.

Proof . For $z \in \mathcal{B}$ call $w \equiv z^{-1}$ the "image" of $z$, and let the open set $\mathcal{B}_{i}$ given by

$$
\mathcal{B}_{i}:=\left\{w \in D_{<1} \text { such that } w^{-1} \in \mathcal{B}\right\}
$$

be the "image set" of $\mathcal{B}$.

From Theorem 3.1 we know that $\tau(w)$ has no zeros on $D_{<1}$. Hence, for $w \in \mathcal{B}_{i},|\tau(w)|>\rho_{1}$, for some positive $\rho_{1}$. Next, we argue that there is also a positive constant $\rho_{2}$ such that $\left|\tau_{w}^{(n)}(1)\right|>\rho_{2}$ for all $n \in \mathbb{N}$ and all $w \in \mathcal{B}_{i}$. For, notice that no element of the sequence of functions $\tau_{w}^{(n)}(1)$ has a zero in $\mathcal{B}_{i}$. Since the sequence converges uniformly and the limit function has no zeros in $\mathcal{B}_{i}$, there must be a positive constant such that $\left|\tau_{w}^{(n)}(1)\right|>\rho_{2}$ for all $w \in \mathcal{B}_{i}$ and $n \in \mathbb{N}$. The desired uniformity follows now from

$$
\left|\tau_{z}^{(n)}-\frac{1}{\tau\left(z^{-1}\right)}\right|=\left|\frac{\tau(w)-\tau_{w}^{(n)}(1)}{\tau(w) \tau_{w}^{(n)}(1)}\right| \leq \frac{1}{\rho_{1} \rho_{2}}\left|\tau(w)-\tau_{w}^{(n)}(1)\right|<\varepsilon
$$

for any prescribed $\varepsilon>0$, uniformly on $\mathcal{B}_{i}$ and for $n$ large enough since, on $\mathcal{B}_{i}$, the sequence $\tau_{w}^{(n)}(1)$ converges uniformly to $\tau(w)$.

Lemma 3.10 Let $\mathcal{B}$ be as in Lemma 3.9. Then the condition

$$
\inf _{z \in \overline{\mathcal{B}}} \inf _{n \in \mathbb{N}}\left|\tau_{z}^{(n)}(1)-\left(\frac{-1}{z \zeta}\right)\right|=0
$$

is impossible for any $\zeta \in(0,1]$.

Proof. By the definition of $\mathcal{B}$, the condition $\tau_{z}^{(n)}(1)+1 /(z \zeta)=0$ does not hold for any finite $n$. Therefore, condition (3.13) says that

$$
\inf _{z \in \overline{\mathcal{B}}}\left|\frac{1}{\tau\left(z^{-1}\right)}-\left(\frac{-1}{z \zeta}\right)\right|=0 .
$$

Since $\overline{\mathcal{B}}$ is closed and $1 / \tau\left(z^{-1}\right)+1 /(z \zeta)$ is a continuous function of $z$ on $\overline{\mathcal{B}}$, this implies the existence of a point $u \in \overline{\mathcal{B}}$ such that $\tau\left(u^{-1}\right)=-u \zeta$. Since $\tau(w)$ fulfills the fixed point equation $\tilde{\tau}=h(w \tilde{\tau})$ for $w \in D_{<1}$, we conclude that $-u \zeta=h(-\zeta) \equiv 0$. But this is impossible for $\zeta>0$ and $u \in D_{>1}$.

Theorem 3.11 (The Second Analyticity Theorem) The function $\mathcal{F}_{r}(z)$ defined in (1.11) is a holomorphic function of $z$ in the open set $D_{>1} \backslash \overline{\mathcal{Z}}$ for all $\mathbf{p} \in \pi$ and $\zeta \in(0,1]$.

If $a_{n} \neq 0$ the singularities of $\mathcal{F}_{r}$ at the points of $\mathcal{Z}_{n}$ are poles of order not greater than $\left(2^{n+1}-1\right) r$.

Proof of Theorem 3.11. Pick an open set $\mathcal{B}$ such that $\overline{\mathcal{B}} \subset D_{>1} \backslash \overline{\mathcal{Z}}$, where $\overline{\mathcal{B}}$ is the closure of $\mathcal{B}$. Then, analyticity of $\mathcal{F}_{r}$ on $\mathcal{B}$ follows from Lemma 3.3 and Lemma 3.10. Since $D_{>1} \backslash \overline{\mathcal{Z}}$ can be covered by such open sets the theorem is proven. 


\subsection{Estimates on the Location of the Poles of $\tau_{z}^{(n)}(1)$}

To study of how fast the sets $\mathcal{Z}_{k}$ accumulate on $S^{1}$ we will make use of a contraction theorem, described below, on the inverse mappings of $h$.

First, some definitions. For $w \in \mathbb{C}, w \neq 1 / \zeta$, define

$$
g(w):=\frac{\zeta-w}{\zeta w-1}
$$

and let $u^{1 / 2}$ be some branch of the square root function in $\mathbb{C}$. Define

$$
h_{+}^{-1}(u):=g\left(u^{1 / 2}\right), \quad h_{-}^{-1}(u):=g\left(-u^{1 / 2}\right) .
$$

Then one has $\left(h \circ h_{ \pm}^{-1}\right)(z)=z, \forall z \in \mathbb{C}$.

Theorem 3.12 (Contraction Theorem) There exists a number $\zeta_{0} \in[0,1 / 3)$, whose approximate value is $\zeta_{0} \simeq 0.29559$, such that for $\zeta \in\left[0, \zeta_{0}\right)$ there exists a strictly positive function e $(\zeta)$ such that for all $u \in D_{1, a(\zeta)}$ with $a(\zeta):=\zeta^{-1}+e(\zeta)$ one has

$$
\left|h_{ \pm}^{-1}(u)\right|<|u| \text {. }
$$

Remark 3.13 Numerical computations indicate to be impossible to improve the region of validity of equation (3.10) to $\zeta \geq \zeta_{0}$.

Remark 3.14 As already observed, the inequality (3.16) becomes an equality in $S^{1}$, which is the internal boundary of $D_{1, a(\zeta)}$. It is important to note also that the set $D_{1, a(\zeta)}$ contains the pole $z=-1 / \zeta$, of $h$.

We will present the proof of the Contraction Theorem in Appendix A. Let us now explore some of its consequences.

Theorem 3.15 Let $\zeta_{0}$ as in Theorem 3.19. For all $\zeta \in\left(0, \zeta_{0}\right)$ and $k \in \mathbb{N}$

$$
\mathcal{Z}_{k} \subset D_{1, r_{k}}
$$

holds with $r_{k}=\zeta^{\frac{-1}{k+1}}$.

This theorem illustrates explicitly that the accumulation points $\mathcal{Z}^{0}$ lie in the unit circle and shows how fast the sets $\mathcal{Z}_{k}$ converge to it, at least for $\zeta \in\left(0, \zeta_{0}\right)$.

Proof. The proof of Theorem 3.15 makes use of the Contraction Theorem which requires the following technical lemma. We note that, from equation (3.11), there exists a finite sequence of signals $\{s(l) \in\{-,+\}, 1 \leq l \leq k\}$ such that

$$
\frac{1}{z} h_{s(k)}^{-1}\left(\frac{1}{z} h_{s(k-1)}^{-1}\left(\frac{1}{z} \cdots \frac{1}{z} h_{s(1)}^{-1}\left(-\frac{1}{z \zeta}\right) \cdots\right)\right)=1 .
$$


Lemma 3.16 Given $z \in \mathcal{Z}_{k}, k \in \mathbb{N}, k \geq 1$, consider a sequence of signals $\{s(l) \in\{-,+\}, 1 \leq l \leq k\}$ satisfying (3.18) and define

$$
w_{l}:=\frac{1}{z} h_{s(l)}^{-1}\left(\frac{1}{z} h_{s(l-1)}^{-1}\left(\frac{1}{z} \cdots \frac{1}{z} h_{s(1)}^{-1}\left(-\frac{1}{z \zeta}\right) \cdots\right)\right), \quad l \in\{1, \ldots, k\} .
$$

Then all $w_{l}$ 's belong to $D_{1, a(\zeta)}$, except, of course, $w_{k}$ which is equal to 1 .

Proof. To prove Lemma 3.16 we take, without loss of generality, $k>1$ and note that, since $z \in D_{1,1 / \zeta}$, one has $h_{s(1)}^{-1}\left(-z^{-1} / \zeta\right) \in D_{1,1 / \zeta}$, by the Contraction Theorem. Hence, $w_{1} \in D_{\zeta, 1 / \zeta}$.

On the other hand $w_{1}$ cannot belong to $D_{\zeta, 1} \cup S^{1}$ for the following reason: $h_{ \pm}^{-1}$ maps $D_{<1} \cup S^{1}$ into itself and, since $1 /|z|<1$, this implies $w_{2} \in D_{<1}$, which, in turn, would imply that all subsequent $w_{l}$ 's would also be in $D_{<1}$ and so they could not reach the point 1 after a finite number of steps.

Therefore, $w_{1} \in D_{1,1 / \zeta}$ and we can apply again the Contraction Theorem and claim that $h_{s(2)}^{-1}\left(w_{1}\right) \in D_{1,1 / \zeta}$. So, we conclude again that $w_{2} \in D_{\zeta, 1 / \zeta}$. If $k=2$ then, by the hypotheses, $w_{2}=1$. Otherwise, if $k>2$, repeating the previous arguments, we conclude again that $w_{2} \in D_{1}, 1 / \zeta$. This argument can be repeated a finite number of times, thus proving the lemma.

Lemma 3.16 implies that all $w_{l}, 1 \leq l<k$, lie in the region of validity of the Contraction Theorem. Therefore, applying the inequality (3.16) repeatedly in (3.18) we get $\zeta|z|^{k+1}<1$, which proves (3.17). The fact that $\mathcal{Z}^{0} \subset S^{1}$ follows easily from (3.17). This completes the proof of Theorem 3.15.

The Contraction Theorem has another consequence.

Theorem 3.17 For the sets $\mathcal{Z}_{k}$ defined above we have, in the region of validity of the Contraction Theorem, that $\mathcal{Z}_{l} \cap \mathcal{Z}_{m}=\emptyset$, for all $l, m \in \mathbb{N}, l \neq m$.

Proof. Let $m>l$ and pick a $z \in \mathcal{Z}_{l} \cap \mathcal{Z}_{m}$. Then one has

$$
\tau_{z}^{(m-l)}\left(-\frac{1}{z \zeta}\right)=-\frac{1}{z \zeta}
$$

This means also that there exists a finite sequence $\{s(a) \in\{-,+\}, 1 \leq a \leq m-l\}$ such that

$$
-\frac{1}{z \zeta}=\frac{1}{z} h_{s(m-l)}^{-1}\left(\frac{1}{z} h_{s(m-l-1)}^{-1}\left(\frac{1}{z} \cdots \frac{1}{z} h_{s(1)}^{-1}\left(-\frac{1}{z \zeta}\right) \cdots\right)\right) .
$$

Applying the Contraction Theorem to the last equality we get

$$
\frac{1}{|z| \zeta}<\frac{1}{|z|^{m-l+1} \zeta}
$$

what means $|z|^{m-l}<1$, a contradiction since $\mathcal{Z}_{l} \cap \mathcal{Z}_{m} \subset D_{1,1 / \zeta}$. 


\section{A The Contraction Theorem}

This appendix is devoted to the proof of Theorem 3.12 which is implied by the following theorem.

Theorem A.1 There exists a $\zeta_{0} \in[0,1 / 3)$, whose approximate value is $\zeta_{0} \simeq 0.29559$, such that there is a strictly positive function $f(\zeta)$ in the interval $\zeta \in\left[0, \zeta_{0}\right)$ such that, in the open set defined by $1<|w|<\frac{1}{\sqrt{\zeta}}+f(\zeta), w \in \mathbb{C}$, one has $|g(w)|<|w|^{2}$.

In view of (3.14), (3.15) and Theorem A.1, for $u \in \mathbb{C}$ such that

$$
1<|u|<\left(\frac{1}{\sqrt{\zeta}}+f(\zeta)\right)^{2}=: \frac{1}{\zeta}+\epsilon(\zeta),
$$

one has $\left|h_{ \pm}^{-1}(u)\right|=\left|g\left( \pm u^{1 / 2}\right)\right|<|u|$. This concludes the proof of Theorem 3.12 .

Proof of Theorem A.1. Writing $w=x+i y$, with $x, y \in \mathbb{R}$, a simple computation shows that

$$
|g(w)|^{2}=\frac{\left(\zeta^{2}+|w|^{2}\right)-2 \zeta x}{\left(1+\zeta^{2}|w|^{2}\right)-2 \zeta x} .
$$

To analyze the right hand side, consider the following lemma.

Lemma A.2 Let $t: \mathbb{R} \longmapsto \mathbb{R}$, be given by $t(a):=(\alpha-\beta a)(\gamma-\beta a)$, for $\alpha, \beta$ and $\gamma \in \mathbb{R}$ with $\alpha>\gamma>0$ and $\beta \geq 0$. Consider the open interval $J:=(-\infty, \gamma / \beta)$. Then, $t$ is a continuous, strictly positive, nowhere constant (for $\beta>0$ ) and increasing function in $J$.

Proof. Continuity on $J$ is obvious. A computation shows that $t^{\prime}(a)=\beta(\alpha-\gamma) /(\gamma-\beta a)^{2}$. All claims follow immediately from continuity and from this relation.

Looking at (A.1) we can make the following identifications:

$$
\alpha \equiv \zeta^{2}+|w|^{2} ; \quad \beta \equiv 2 \zeta ; \quad \gamma \equiv 1+\zeta^{2}|w|^{2} ; \quad a \equiv x .
$$

Note that, in view of this

$$
\alpha-\gamma=\left(1-\zeta^{2}\right)\left(|w|^{2}-1\right)>0,
$$

for all $w \in D_{>1}$. Moreover, the condition $a<\gamma / \beta$ means

$$
x<\frac{1+\zeta^{2}|w|^{2}}{2 \zeta}
$$

This condition is always satisfied if $|w|<1 / \zeta$, since $x \leq|w|$ and since the stronger condition

$$
|w|<\frac{1+\zeta^{2}|w|^{2}}{2 \zeta}
$$


is equivalent to the condition $(1-\zeta|w|)^{2}>0$.

In view of Lemma A.2 we conclude that, for $w \in D_{1,1 / \zeta}$,

$$
|g(w)|^{2} \leq \frac{\zeta^{2}+|w|^{2}-2 \zeta|w|}{1+\zeta^{2}|w|^{2}-2 \zeta|w|}=\frac{(\zeta-|w|)^{2}}{(1-\zeta|w|)^{2}}
$$

Finally, note that $D_{1,1 / \sqrt{\zeta}+f(\zeta)} \subset D_{1,1 / \zeta}$ for a convenient $f$.

Now we come to the technically most important result of this appendix.

Theorem A.3 Under the assumptions of Theorem A.1 one has

$$
\frac{(\zeta-|w|)^{2}}{(1-\zeta|w|)^{2}}<|w|^{4}
$$

Proof. Condition (A.4) is equivalent to the condition that the polynomial

$$
P(t):=(\zeta-t)^{2}-t^{4}(1-\zeta t)^{2}
$$

is strictly negative for $1<t<\frac{1}{\sqrt{\zeta}}+f(\zeta)$. Writing it explicitly one has

$$
\begin{aligned}
P(t) & =-\zeta^{2} t^{6}+2 \zeta t^{5}-t^{4}+t^{2}-2 \zeta t+\zeta^{2} \\
& =\left((\zeta-t)+t^{2}(1-\zeta t)\right)\left((\zeta-t)-t^{2}(1-\zeta t)\right) .
\end{aligned}
$$

Using the fact that \pm 1 is a root of the polynomial $(\zeta-t) \pm t^{2}(1-\zeta t)$, we can write

$$
P(t)=(t-1)(t+1)\left(-\zeta t^{2}+(1-\zeta) t-\zeta\right)\left(\zeta t^{2}-(1+\zeta) t+\zeta\right) .
$$

This last relation allows us to find explicitly the roots of $P(t)$ : they are the elements of the set $\left\{-1,+1, a_{-}, a_{+}, b_{-}, b_{+}\right\}$, where

$$
a_{ \pm}:=\left(\frac{1-\zeta}{2 \zeta}\right) \pm \frac{1}{2 \zeta} \sqrt{(1+\zeta)(1-3 \zeta)}
$$

and

$$
b_{ \pm}:=\left(\frac{1+\zeta}{2 \zeta}\right) \pm \frac{1}{2 \zeta} \sqrt{(1-\zeta)(1+3 \zeta)} .
$$

The following lemma gives the possible signs of the polynomial $P(t)$ on the real axis.

Lemma A.4 For $a_{ \pm}$and $b_{ \pm}$defined above as functions of $\zeta$, one has the following results:

1. For $0 \leq \zeta<1 / 3$ one has $0 \leq a_{-} \leq 1$.

2. For $0 \leq \zeta<1$ one has $0 \leq b_{-} \leq 1$. 
3. For $0 \leq \zeta<1 / 3$ one has $b_{+}-a_{+} \geq 1$.

4. For $0 \leq \zeta<\zeta_{0}$ one has $a_{+}-1 / \sqrt{\zeta}>0$, where $\zeta_{0} \in[0,1 / 3), \zeta_{0} \simeq 0.29559$. This in particular says that $a_{+}>1$ and, by $3 ., b_{+}>1$.

Before we prove this lemma, let us finish the proof of Theorem A.3 and, hence, of the Theorem A.1. According to Lemma A.4, $P(t)<0$ if $1<t<a_{+}$. This follows from the localization of the roots and from the fact that $P$ is a polynomial of even degree with a negative leading term. See Figure 3 .

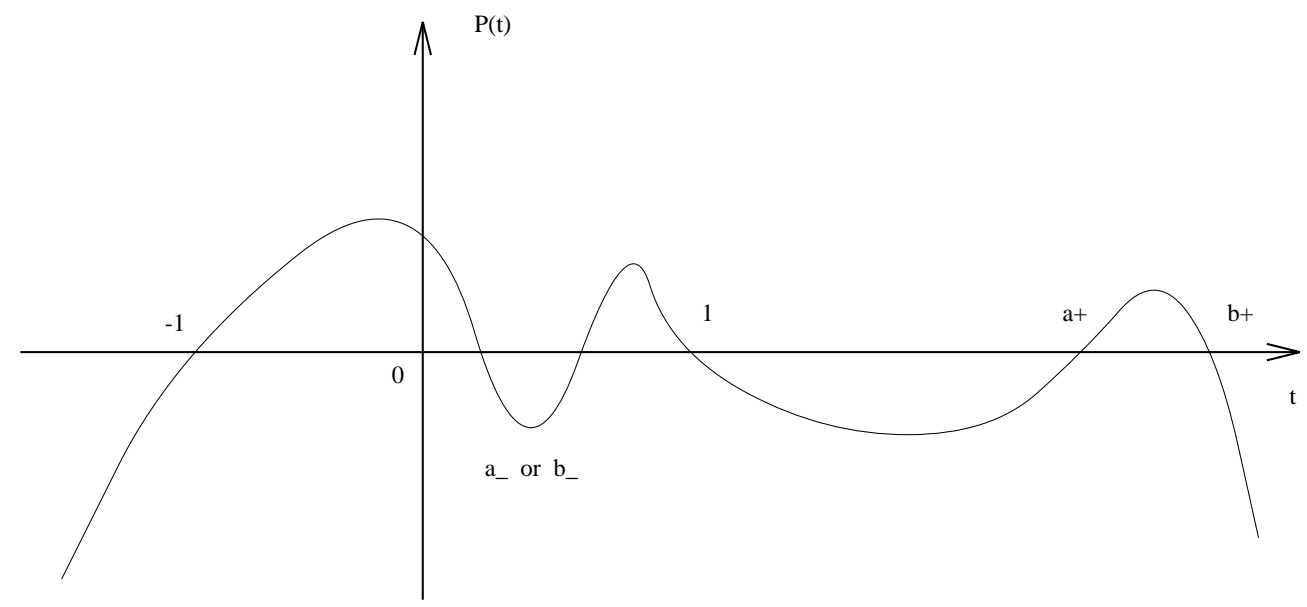

Figure 3: The graphic of $P(t)$.

The proof if completed by defining $f(\zeta):=a_{+}(\zeta)-1 / \sqrt{\zeta}$, which, according with item 4 of Lemma A.4, is strictly positive for $0 \leq \zeta<\zeta_{0}$. This completes also the proof of the Contraction Theorem.

Proof of Lemma A.4. We will prove each of the items of Lemma A.4 separately.

\section{Proof of item 1.}

The hypothesis that $a_{-} \geq 0$ is equivalent to $(1-\zeta)^{2} \geq(1+\zeta)(1-3 \zeta)$ for $0 \leq \zeta<1 / 3$. This last relation means $4 \zeta^{2} \geq 0$, which is obviously verified.

The hypothesis that $a_{-} \leq 1$ is equivalent to

$$
1-\sqrt{(1+\zeta)(1-3 \zeta)} \leq 3 \zeta
$$

which is equivalent to $(1-3 \zeta)^{2} \leq 1-2 \zeta-3 \zeta^{2}$. This means $4 \zeta(3 \zeta-1) \leq 0$, what is true for $0 \leq \zeta<1 / 3$, the equality holding only if $\zeta=0$.

Proof of item 2.

The hypothesis that $b_{-} \geq 0$ is equivalent to $(1+\zeta)^{2} \geq(1-\zeta)(1+3 \zeta)$, which is equivalent to $4 \zeta^{2} \geq 0$, which is, of course, always true. 
The hypothesis that $b_{-} \leq 1$ is equivalent to $(1-\zeta)^{2} \leq(1-\zeta)(1+3 \zeta)$. This means that $4 \zeta(\zeta-1) \leq 0$, which is always true for $0 \leq \zeta \leq 1$.

Proof of item 3.

Since

$$
b_{+}-a_{-}=1+\frac{1}{2 \zeta}\left(\sqrt{1+2 \zeta-3 \zeta^{2}}-\sqrt{1-2 \zeta-3 \zeta^{2}}\right)
$$

item 3 is proven, provided the term between parenthesis above is positive. This is implied by the inequality $1+2 \zeta-3 \zeta^{2} \geq 1-2 \zeta-3 \zeta^{2}$, which is always true for $\zeta \geq 0$.

Proof of item 4 .

The condition $a_{+}>1 / \sqrt{\zeta}$ means that

$$
\sqrt{(1+\zeta)(1-3 \zeta)}>\zeta+2 \sqrt{\zeta}-1
$$

The right hand side is strictly negative for $0 \leq \zeta<(\sqrt{2}-1)^{2} \simeq 0.171$. So, in this region the condition above is automatically satisfied. On the other hand, if the right hand side of (A.6) is positive, we can square both sides and arrive to the equivalent condition

$$
4 s\left(s^{3}+s^{2}+s-1\right)<0
$$

where $s=\sqrt{\zeta}$. The polynomial $s^{3}+s^{2}+s-1$ has one real root at $s_{0} \simeq 0.543689$ and two complex roots at $s_{ \pm} \simeq-0.77 \pm 1.115 i$. We call $\zeta_{0}:=s_{0}^{2}$, which gives $\zeta_{0} \simeq 0.295597$. Thus, condition (A.7) is satisfied for $0<\zeta<\zeta_{0}$. With this the proof of Lemma A.4 is complete.

\section{References}

[BM] J C A Barata and D H U Marchetti. "Griffiths' Singularities in Diluted Ising Models on the Cayley Tree". To appear in J. Stat. Phys. 88, (1997).

[G] Robert B Griffiths. "Nonanalytic Behavior Above the Critical Point in a Random Ising Ferromagnet". Phys. Rev. Lett. 23, 17-19 (1969).

[P] J F Perez. "Controlling the Effect of Griffiths' Singularities in Random Ferromagnets: Smoothness of the Magnetization". Brazilian Journal of Physics 23, 356-362 (1993).

[Fe] William Feller. "An Introduction to Probability Theory and its Applications", Vol. 2. John Wiley \& Sons. Second edition, 1971.

[T] E C Titchmarsh. "The Theory of Functions". Oxford University Press. Second Edition, 1939. 